\title{
A Numerical Analytical Method of Parameter Optimization for Fast-starting of the Bionic Robot Fish
}

\author{
http://dx.doi.org/10.3991/ijoe.v9iS6.2791 \\ Chao Yang \\ Jiangsu University of Technology, Changzhou, China
}

\begin{abstract}
In this study, the alloy sheet,the giant magnetostrictive material (GMM), was stuck on the sheet, and the external magnetic field were simulated as the framework of the fishtail, respectively; the muscle of the fish and the nerve are to control the swing of the fishtail for simulating the fast-starting of some natural fishes. Simulate the Faststarting of fish different environment of nature. A mechanics model and new numerical method are presented by analyzing the vibrational modes. Optimization for External Parameter was conducted by using numerical computation, the best environment and material for Faststarting of the Bionic Robot fish is revealed. Results show that the suit material can help the robot fish complete the fast-transition from the lower order mode to the higher order mode. The method and results provide theoretical foundation and applies basis to designing bionic robot fish. Do not cite references in the abstract.
\end{abstract}

Index Terms-Bionic robot fish, Cantilever interlayer sheet, GMM,Fast-start performance.

\section{INTRODUCTION}

With the development of modern science, it becomes more and more urgent to access a new mini-tube robot [1] which can move stably and flexibly in water or watermedium in medical domain and industry application, including bionic robot fish of GMM [2-4]. The robot can be driven wirelessly in a tiny room, such as in people's vessel.

Quick start-up can be divided into C shape start and S shape start by swing morphology of fish when fish starts. Different start modes are formed due to different rigidity of fishtail and natural surroundings such as liquid surroundings. For example, Northern Pike can start instantly, and its maximum acceleration is 25 times of acceleration of gravity [5]. Several countries have conducted study on the way bionic robot fish starts to reveal the principle. For example, bionic robot tuna and bionic barracuda developed by MIT are driven by motor. American Naval Weapon Laboratory discovered shape memory alloy in 1963, realizing SMA driven robot. Professor Toshio Fukuda in Nagoya University developed mini underwater mobile robot, and it utilizes piezoelectric ceramics $(\mathrm{Pb}(\mathrm{Zr}, \mathrm{Ti}) \mathrm{O} 3)$ to drive the swing of two symmetric legs to move it. Festo developed a kind of pneumatically artificial muscle called pneumatic tendon MAS, and a swing structure consists of two tendons is developed. The disadvantages of driving system above are that they're wired or with power plant. A mini robot will overcome two disadvantages above to be driven by external field if it can absorb energy from a certain external field to obtain sufficient energy.

GMM is a new kind of function materials developed in 1970 s. It is regarded as a strategic function materials related to improve comprehensive competitiveness of national high technology in 21 century. The emergence and development of GMM provides a better choice to utilize no-cable control and design special mini robot fish. The study chooses GMM as fishtail materials. Robot fish is controlled through external magnet field in this study. Then the $\mathrm{S}$ shape start and $\mathrm{C}$ shape start are revealed through relative principles obtained by optimizing fish material and external surroundings parameters. The study provided the foundation of quick start-up [6] of bionic robot fish $[7,8]$.

\section{DRIVING AND CONTROL EQUATION OF BIONIC ROBOT FISH}

Bionic robot fish of GMM consisted of fish body and fishtail, and the fishtail is formed from pasting or plating giant magnetostrictive material. The change of bulk and size of giant magnetostrictive material under the changeable external magnet can lead to a dynamic loading distribution of alloy sheet, driving the fishtail to swing.

\section{A. structure model of bionic robot fish}

Figure 1 shows that Cartesian coordinate is utilized, and the length is $\mathrm{L}$, the width is $\mathrm{b}$ and the height is $\mathrm{h}$. The thickness $\xi(x)$ of giant magnetostrictive material by pasting or plating (the width of the sheet remains unchanged) is thinner than alloy sheet, namely $\xi(x) \prec h$.

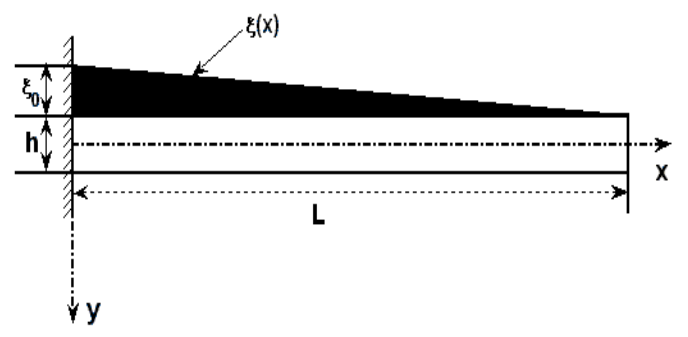

Figure 1. Cantilever interlayer plate properties and coordinates

According to the study by Clark [9](1980), the constitutive relation of flexibility of giant magnetostrictive material and external magnet parameter can be represented by 


$$
\varepsilon=\varepsilon_{\sigma}+\varepsilon_{H}=S^{H} \sigma+d H
$$

Where, $\varepsilon$ represents overall strain. $S^{H}$ is compliance influence coefficient. $\sigma$ is stress.d is dynamic magnetostrictive coefficient. $\mathrm{H}$ is external magnet intensity.

The strain caused by external magnetic field intensity $\varepsilon_{H}$ is

$$
\varepsilon_{H}=\frac{\Delta L}{L}=\lambda=d H
$$

$\lambda$ stands for magnetostrictive coefficient. The material has both magnetism and elasticity, and it can degenerate to single character. For instance, when considered pure elastic material, let $\varepsilon_{H}=0$ and $S^{H}=1 / E$ (E is elasticity modulus). The relation between intensity of external and magnet induction intensity $\mathrm{B}$ is in linear scale. It is assumed that the magnet induction intensity $\mathrm{B}$ is change exponentially. So

$$
H=\frac{B}{\mu}=B \cos (\Omega t)=B_{0}=\left(1+e^{-\varepsilon t}\right) \cos (\Omega t)
$$

$\mathrm{B}_{0}$ and $\Omega$ are amplitude of external magnet induction intensity and frequency of magnet field, respectively, and $\mu$ is the permeability. Giant magnetostrictive material pasted or plated in the elastic sheet is flexible with the change of external magnet field. The effect produces on sheet can be concluded a shear force distribution and finally transferred to bending moment distribution.

$$
\begin{aligned}
& G(x, t)=\int_{A} \sigma y d A= \\
& \int_{h / 2}^{\xi(x)+h / 2} E_{2} d b B_{0}\left(1+e^{-\varepsilon t}\right) \cos (\Omega t) y d y
\end{aligned}
$$

$b$ is width of fishtail. The thickness of giant magnetostrictive material is $\xi(x)=\xi_{0}(1-x / L), \xi_{0}$ is a constant.

\section{B. The mechanism of bionic robot fish}

The problem can be simplified to a cantilever beam model by ignoring the influence of giant magnetostrictive material. It is assumed that the fishtail doesn't reverse or transform, and the liquid resistance the fishtail suffers is approximately the same as the resistance generated in the flow field $[10,11]$. Referring to the resistance given by Gerhart [12] etc., in low Reynolds number, the damping coefficient [11] $\mathrm{C}$ can be approximated to $C=\mu_{0} \pi^{2} L / 2[\ln (2 L / b)-0.5+\ln 2]$, in which $\mu_{0}$ is the viscosity coefficient of liquid.

The control equation of fishtail swing is

$$
E I \frac{\partial^{4} y(x, t)}{\partial x^{4}}+\rho b h \frac{\partial^{2} y(x, t)}{\partial t^{2}}+C \frac{\partial y(x, t)}{\partial t}=\eta(t)
$$

When the giant magnetostrictive material is linearly distributed, $\eta(t)=\gamma\left(1+e^{-\varepsilon t}\right) \cos (\Omega t)$, in which $\gamma=-E_{2} b d B_{0}\left(1+e^{-\varepsilon t}\right)\left(\xi_{0} / L\right)^{2}$. The corresponding boundary conditions are

$$
\left\{\begin{array}{l}
\left.y(x, t)\right|_{x=0}=0,\left.\frac{\partial y(x, t)}{\partial x}\right|_{x=0}=0 \\
\left.\frac{\partial y^{2}(x, t)}{\partial x^{2}}\right|_{x=L}=0,\left.\frac{\partial y^{3}(x, t)}{\partial x^{3}}\right|_{x=L}=0
\end{array} .\right.
$$

Initial conditions are

$$
y(x, 0)=y_{0}, \dot{y}(x, 0)=\dot{y}_{0}(x)=0
$$

Incorporate the control equation (5) with initial conditions to obtain $E I y_{0}{ }^{(4)}=2 \gamma$ and integrated as follows:

$$
y_{0}=2 \gamma\left(\frac{x^{4}}{24}+c_{1} \frac{x^{3}}{6}+c_{2} \frac{x^{2}}{2}+c_{3} x+c_{4}\right) / E I
$$

Incorporate boundary conditions (6) and initial conditions (7) with equation (8) $c_{3}=c_{4}=0, c_{1}=-L, c_{2}=L^{2} / 2$, let $\mathrm{y}=\mathrm{y} 0+\mathrm{y} 1$ incorporate with control equation (5):

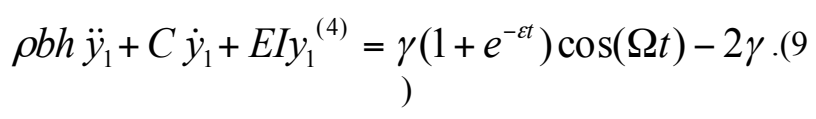

Initial conditions are transferred to $y_{1}(x, 0)=0, \dot{y}_{1}(x, 0)=0$. The solution of equation consists of general solution $y_{c}$ and particular solution $y_{p}$, and let $y_{1}=y_{c}+y_{p}$. The particular solution can be resolved into the summation of two following equations.

$$
\rho b h \ddot{y}_{p 1}+C \dot{y}_{p 1}+E I y_{p 1}{ }^{(4)}=-2 \gamma
$$

$$
\rho b h \ddot{y}_{p 2}+C \dot{y}_{p 2}+E I y_{p 2}{ }^{(4)}=\gamma\left(1+e^{-\varepsilon t}\right) \cos (\Omega t)
$$

$y_{p 1}=-y_{0}$ can be obtained by observing equation (10), thus,

$$
y=y_{0}+y_{1}=y_{0}+y_{c}+y_{p 1}+y_{p 2}=y_{c}+y_{p 2} \text {. }
$$




\section{SOLUTION METHOD OF CONTROL EQUATION}

A. Natural frequency of system [13]

The natural frequency can be expressed as $\omega_{j}=k_{j}^{2} \sqrt{E I / \rho b h} \quad(j=1,2,3 \ldots) \quad, \quad$ and $\quad$ the corresponding vibration function can be expressed as

$$
\begin{gathered}
Y_{j}(x)=\operatorname{ch}\left(k_{j} x\right)-\cos \left(k_{j} x\right)-\frac{\cos \left(k_{j} L\right)+\operatorname{ch}\left(k_{j} L\right)}{\sin \left(k_{j} L\right)+\operatorname{sh}\left(k_{j} L\right)} \\
*\left[\operatorname{sh}\left(k_{j} x\right)-\sin \left(k_{j} x\right)\right] \quad(j=1,2,3 \ldots)
\end{gathered}
$$

The factor $\lambda_{j}=1 /\left\{\int_{0}^{L}\left[Y_{j}(x)\right]^{2} d x\right\}^{1 / 2}$ is multiplied to the former equation for normalization. The vibration functions have the flowing orthogonal relationship.

$$
\begin{aligned}
& \int_{0}^{L} Y_{i}(x) \cdot Y_{j}(x) d x=\delta_{i j} \\
& \int_{0}^{L} Y_{i}^{(4)}(x) \cdot Y_{j}(x) d x=k_{i}^{4} \cdot \delta_{i j}
\end{aligned}
$$

\section{B. The solution of control equation}

The variables separation method $y(x, t)=\phi(t) Y(x)$ [13] is utilized to obtain the solution of equation (11), namely.

$$
y(x, t)=\sum_{i}^{\infty} \phi_{i}(t) Y_{i}(x)
$$

After substituted by equation (14), equation (11) is multiplied by $Y_{j}(x)$, and the result is integrated along the whole beam. Then orthogonality of vibration function is introduced as follows:

$$
M_{i} \ddot{\phi}_{i}(t)+C_{i} \dot{\phi}_{i}(t)+K_{i}(t) \phi_{i}(t)=\gamma_{i}(t)
$$

Where $M_{i}=\rho b h \int_{0}^{L} Y_{i}^{2}(x) d x, C_{i}=C \int_{0}^{L} Y_{i}^{2}(x) d x$, $\gamma_{i}(t)=\gamma\left(1+e^{-\varepsilon t}\right) \cos (\Omega t) \int_{0}^{L} Y_{i}^{2}(x) d x$

$K_{i}=\int_{0}^{L} E I Y_{i}(x) Y_{i}^{(4)}(x) d x$. The particular solution of equation (15) is

$$
\begin{gathered}
\phi_{i}^{(p)}(t)=a_{1 i} \cos (\Omega t)+b_{1 i} \sin (\Omega t)+ \\
e^{-\varepsilon t}\left[a_{2 i} \cos (\Omega t)+b_{2 i} \sin (\Omega t)\right]
\end{gathered}
$$

$$
\begin{aligned}
& \text { where } a_{1 i}=-\bar{m} \gamma A_{i}\left(\Omega^{2}-\omega_{i}{ }^{2}\right) /\left[B _ { i } \left(\bar{m}^{2} \Omega^{4}-\right.\right. \\
& \left.\left.2 \bar{m}^{2} \Omega^{2} \omega_{i}{ }^{2}+C^{2} \Omega^{2}+\bar{m}^{2} \omega_{i}{ }^{4}\right)\right], b_{1 i}=-\Omega C \gamma A_{i} / \\
& {\left[B_{i}\left(\bar{m}^{2} \Omega^{4}-2 \bar{m}^{2} \Omega^{2} \omega_{i}{ }^{2}+C^{2} \Omega^{2}+\bar{m}^{2} \omega_{i}{ }^{4}\right)\right]} \\
& a_{2 i}=\gamma A_{i} \lambda_{1 i} /\left[\bar{m} B_{i}\left(\lambda_{1 i}{ }^{2}+\lambda_{2 i}{ }^{2}\right)\right] \\
& , \quad\left[\bar{m} B_{i}\left(\lambda_{1 i}{ }^{2}+\lambda_{2 i}{ }^{2}\right)\right] \\
& \lambda_{1 i}=\varepsilon^{2}-\Omega^{2}-C \varepsilon / \bar{m}+\omega_{i}{ }^{2}, \lambda_{2}=C \Omega / \bar{m}-2 \varepsilon \Omega \\
& \omega_{i}^{2}=K_{i} / M_{i}, \\
& A_{i}=\int_{0}^{L} Y_{i}(x) d x, B_{i}=\int_{0}^{L} Y_{i}{ }^{2}(x) d x, \bar{m}=\rho b h . \text { The }
\end{aligned}
$$
general solution of equation (16) $\bar{\phi}_{i}$ can be obtained

$$
\begin{cases}\bar{\phi}_{i}(t)=c_{1 \mathrm{i}} e^{r_{1} t}+c_{2 i} e^{r_{2} t} & \Delta_{\mathrm{i}}>0 \\ \bar{\phi}_{i}(t)=\left(c_{1 i}+c_{2 i} t\right) e^{\alpha t} & \Delta_{i}=0 .(17) \\ \bar{\phi}_{i}(t)=e^{\alpha t}\left(c_{1 i} \cos \left(\boldsymbol{\beta}_{i} t\right)+c_{2 i} \sin \left(\boldsymbol{\beta}_{i} t\right)\right) & \Delta_{i}<0\end{cases}
$$$$
\text { where } \Delta_{i}=(C / \bar{m})^{2}-4 \omega_{i}^{2}, \gamma_{1,2(i)}=-[C / \bar{m}
$$$$
\pm \sqrt{\Delta_{i}} / 2 \quad, \quad \alpha=-C /(2 \bar{m}), \beta_{i}=\sqrt{-\Delta_{i}} / 2 \quad \text {; }
$$

$c_{1 i}=\left[\left(\mathrm{z}_{i} / B_{i}-a_{1 i}-a_{2 i}\right) r_{2}-\boldsymbol{\varepsilon} a_{2 i}+b_{2 i} \Omega+b_{1 i} \Omega\right] /\left(r_{2}-r_{1}\right)$,

$\mathrm{c}_{2 i}=\left[\left(\mathrm{z}_{i} / B_{i}-a_{1 i}-a_{2 i}\right) r_{1}-\varepsilon a_{2 i}+b_{2 i} \Omega+b_{1 i} \Omega\right] /\left(r_{1}-r_{2}\right) \Delta_{\mathrm{i}}>0$

$\mathrm{c}_{1 i}=\mathrm{z}_{i} / B_{i}-a_{1 i}-a_{2 i}, c_{2 i}=\boldsymbol{\varepsilon} a_{2 i}-b_{1 i} \Omega-b_{2 i} \Omega-$

$\alpha\left(\mathrm{z}_{i} / B_{i}-a_{1 i}-a_{2 i}\right) \Delta_{i}=0, \quad \mathrm{c}_{1 i}=\mathrm{z}_{i} / B_{i}-a_{1 i}-$

$a_{2 i} \quad, \quad \mathrm{c}_{2 i}=\left[\boldsymbol{\varepsilon} a_{2 i}-b_{1 i} \Omega-b_{2 i} \Omega-\boldsymbol{\alpha}\left(\mathrm{z}_{i} / B_{i}-a_{1 i}\right.\right.$

$\left.\left.-a_{2 i}\right)\right] / \boldsymbol{\beta}_{i} \quad \Delta_{i}<0$. Thus, the solution of the equation can be expressed as

$y(x, t)=\sum_{i}^{\infty} \phi_{i}(t) Y_{i}(x)=\sum_{i}^{\infty} Y_{i}(x)\left[\phi_{i}^{*}(t)+\bar{\phi}_{i}^{*}(t)\right] .(18)$

\section{Driving force of fishtail swing}

During a magnetic field change interval, the average driving force generated by fishtail swing can be expressed as

$$
\begin{gathered}
F_{a d}=\frac{1}{t_{3}} \int_{0}^{t_{3}} F(t) d t=\frac{1}{t_{3}} \int_{0}^{t_{3}} \int_{0}^{L} C \dot{y}(x, t) . \\
y^{\prime}(x, t) d x d t
\end{gathered}
$$

\section{NUMERICAL RESULT AND ANALYSIS}

To study the problem quantitatively, the following parameters are chosen. $\xi_{0}=1.0 \times 10^{-5}[\mathrm{~m}], b=1.0 \times 10^{-2}[\mathrm{~m}]$ and $h=1.0 \times 10^{-4}[\mathrm{~m}]$. Aluminum alloy material is utilized in fishtail, and $\mathrm{TbFe}_{2}$ is utilized in giant magnetostrictive material [9]. The first five order modal are superposed. 
The optimal length [3] in stable state is chosen to represent fishtail length $(1=0.09)$. Time during the third time the tail passing through the horizontal axis is chosen as start time.

\section{A. The effect of viscosity coefficient of liquid force on robot fish starting}

Force viscosity coefficient $\mu_{0}$ is set as 1.0 [Pa.s], $1.5[\mathrm{~Pa} . \mathrm{s}]$ and 2.0[Pa.s] for analyzing the effect on start of robot fish under fluid environment. Figure 2 shows the comparison of three kinds of viscosity coefficient about the relationship between external magnetic frequency and average propulsion.

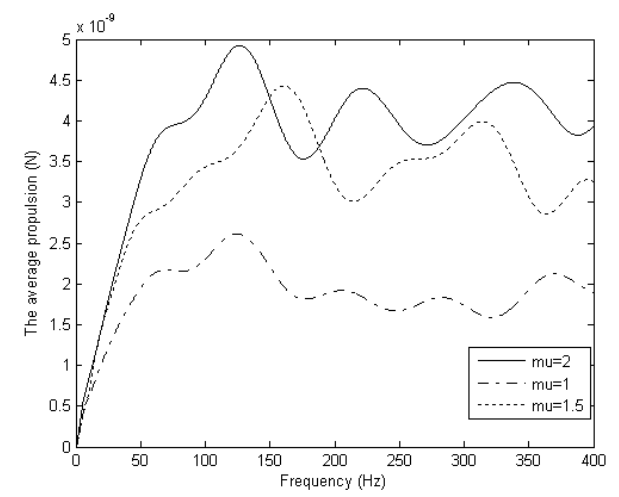

Figure 2. Relations between frequency and average propulsion

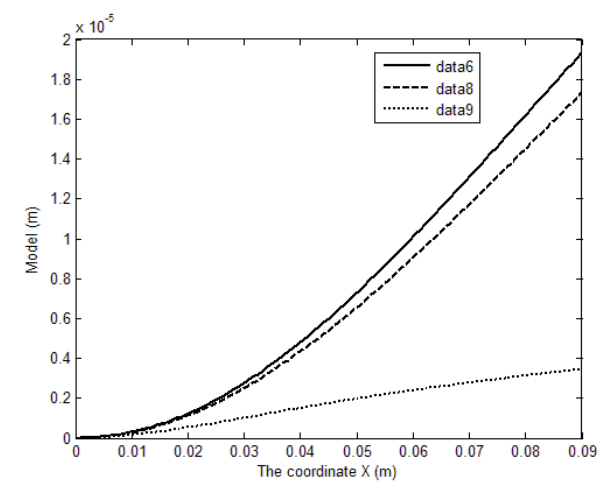

Figure 3. Shapes of the fishtail under the external magnetic fields

Figure 2 shows the average propulsion is maximum when the viscosity coefficient is 2.0 , because the bigger viscosity coefficient leads to the bigger resistance when robot fish move. The fish start shape is showed below with the viscosity coefficient of 1.0(Figure 3), 1,5 (Figure 4) and 2.0(Figure 5).

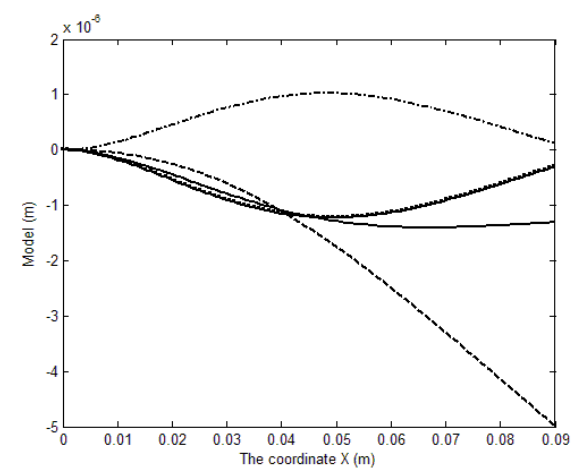

Figure 4. Shapes of the fishtail under the external magnetic fields
Figure 3 - Figure 5 show that the robot fish is in $\mathrm{C}$ shape mode, such as crucian in fresh water, under the external frequency corresponding to maximum average propulsion when $\mu_{0}=1.0$, and the modality of robot fish start exactly transits from low order modality to high order when $\mu_{0}=1.5$. The modality of fishtail swing also change from low order modality to high order when $\mu_{0}=2.0$. It illustrates that the transition from low order modality to high order is crucial for the start of robot fish in the whole process. It is also showed from the figure that the bigger the viscosity coefficient of liquid when fishtail swing, the easier to realize the transform from low order modality to high order. It is the reason that loach transits faster to $\mathrm{S}$ shape mode when starting in mud.

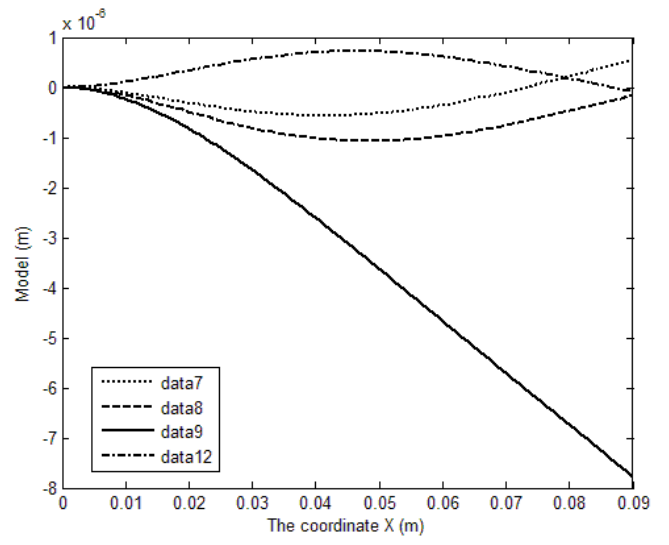

Figure 5. Shapes of the fishtail under the external magnetic fields

\section{B. Influence of fishtail material}

$\mu_{0}=2.0$ is chosen as the dynamic viscosity coefficient of liquid. Aluminum alloy and alloy steel material that is relativelyhard $\left(E=2.0 \times 10^{11}[\mathrm{pa}] \rho=7.85 \times 10^{3} \mathrm{~kg} / \mathrm{m}^{3}\right)$ are selected to compare with the skeleton of fish. The relation between average driving force and frequency of external magnet field is shown in Figure 6.

Figure 6 shows the max average driving force of fishtail made from alloy steel is much smaller than fishtail made of aluminum. This illustrates that the relatively hard fishtail material is unfavorable to small robot fish. In the frequency of external magnet field corresponding to max average driving force, the swing mode of robot fish doesn't transit from low order modality to high order modality. Its start is in C shape. Meanwhile, the fishtail of robot fish made of aluminum alloy material transit from low modality to high order modality in a not very big frequency of external magnet field, namely S shape start, and it can obtain bigger average driving force.

Figure 7 shows different swing modality when fish swims in nature. Turning amplitude of fishtail in the far right of the figure is the smallest, reflecting the swing mode in which the rigidity is relatively big, and the viscosity coefficient is relatively small. Turning amplitude of fishtail in the far left of the figure is the biggest, reflecting the swing mode in which the rigidity is relatively small, and the viscosity coefficient is relatively big. This also verifies the rationality of the model in the study. 


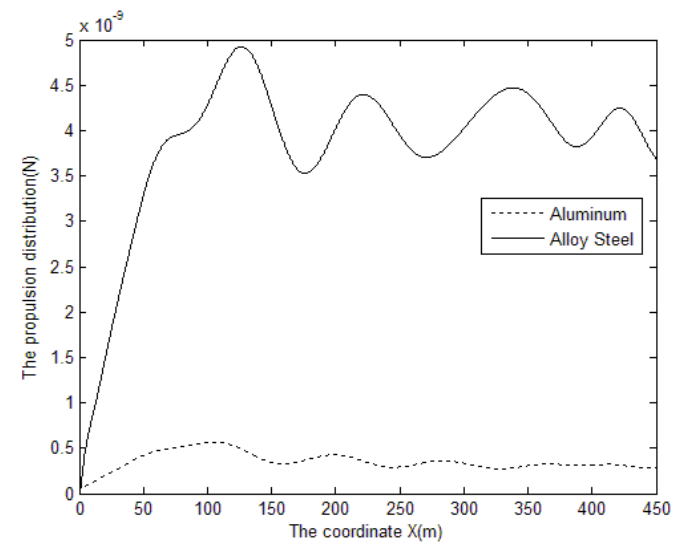

Figure 6. Relations between frequency and average propulsion
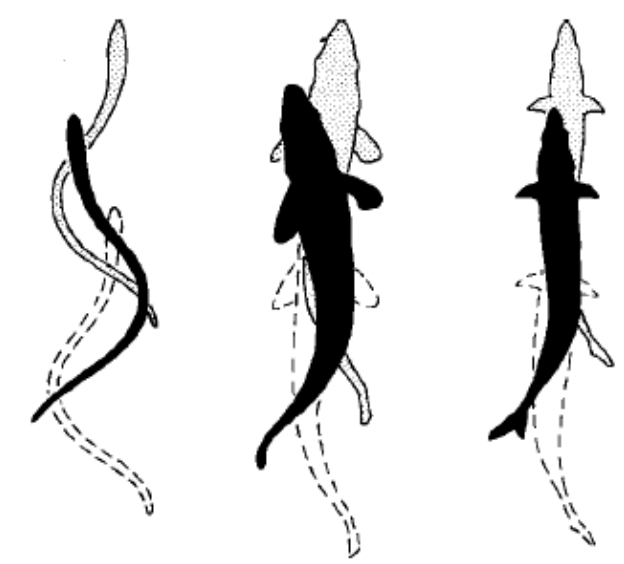

Figure 7. Swimming experiment of fish-like underwater robot

\section{CONCLUSION}

The start speed of bionic robot fish of giant magnetostrictive material can be adjusted by choosing proper fluid environment and fishtail materials. The startup speed of bionic robot fish is related to material constants, geometric parameters, fluid environment and the frequency and intensity of external magnet field. It can make the robot fish successfully transit from low order modality to high order modality to choose proper parameters when it starts. The driving force is closely related to the intensity of external magnet field. The study results verify the rationality of S shape start of fish, and provide evidence for the design on bionic robot fish's quick start-up.

\section{REFERENCES}

[1] Otsuka A. Development of an eating function support system [C]. Proc.1st IARP Workshop Medical and Healthcare Robots, Ottawa, Canada, June 1988. 789-792.

[2] Sun FM, Xu XS, et al. Non-linear Vibration and Dynamic Characteristic of Fish-like Robot Controlled by GMM Actuator. Journal of Intelligent Material Systems and Structures. 20(2009) 1503-1513. http://dx.doi.org/10.1177/1045389X09105234

[3] Sun FM, Xu XS, The Control Mechanism of a New Fish-Like Underwater Robot with Two Tails, Lecture Notes in Computer Science, 2008, LNAI 5314(1) 304-313.

[4] Xu XS, Sun FM, Wang GP, The control and optimization design of the fish-like underwater robot with the aid of the giant magnetostrictive material actuator. Journal of Vibration and Control 2009,15(10): 1443-1462. http://dx.doi.org/10.1177/ 1077546308097264

[5] Schriefer J E, Hale ME. Strikes and startles of northern pike (Esox lucius): a comparison of muscle activity and kinematics between S- start behaviors [J]. J Exp. Biol., 2004, 207: 535- 544. http://dx.doi.org/10.1242/jeb.00789

[6] CHEN Hong,ZHU Chang-an,YIN Xie-zhen,XING Xiao-zheng. Research on fast-start performance of fish-like underwater robot. Journal of Ship Mechanics.2007, 11(5):647-654.

[7] Guo S X, Fukuda T, Asaka K. A new type of fish-like underwater microrobot [J]. IEEE Transactions on Mechatronics. 2003, 8(1) : 136-141.

[8] Saotome H, Okubo T, Ikeda Y. A novel actuator with Nd-Fe-B magnets swimming in parallel to the magnetic field [J]. IEEE Transactions on Magnetics, 2002, 38(5) : 3009-3011. http://dx.doi.org/10.1109/TMAG.2002.803184

[9] Clark A E. Ferromagnetic Material 1 [M]. Wohlfath E P, NorthHolland Publishing Company. 1980.

[10] JI Heng-teng, FAN Ju, HUANG Xiang-lu. Numerical Simulation of Hydrodynamic Forces of Heave Plate. Journal of Shanghai Jiaotong University [J]. 2003, 37(8): 1266-1270.

[11] Prislin I, Blevins R D, Halkyard J E. Viscous damping and added mass of so lid square plates [C]. Proceedings of the International Conference on Offshore Mechanics and Arctic Engineering. ASME Fairfield NJUSA, 1998, 5-9.

[12] Gerhart PM, Gross RJ, Hochstein JI. Fundamentals of Fluid Mechanics [M]. 2nd Edition, Addison Wesley, 1992.

[13] Zhang Yahui,Lin Jiahao. Fundamentals of Structural Dynamics [M]. Dalian University of Technology Press, dalian, 2007.

\section{AUTHORS}

Chao Yang is with School of Materials Engineering, Jiangsu University of Technology, No. 1801, Wuzhong Avenue, Changzhou, Jiangsu Province. 213001 CHINA (e-mail: yc@jstu.edu.cn)

This work was supported in part by the funds of Jiangsu University of Technology (KYY11099). It is an extended and modified version of a paper presented at the 2012 International Conference on Artificial Intelligence and Its Application in Industry Production (AIAIP 2012), held in Wuhan, China in December 2012. Manuscript received 02 May 2013. Published as submitted by the author 26 June 2013. 